\title{
Hypokalaemic Periodic Paralysis
}

\author{
Dr. Anjan K Lenkalapally, Dr. Vikram G Aarella, Dr. Ramya Parasa, Dr. Mahesh Cheryala, Dr. \\ Bhavani Merugu.
}

\section{Introduction}

Hypokalaemic periodic paralysis is a medical emergency when patients present with acute onset paraparesis usually noticed in the mornings secondary to low serum potassium levels with a prevalence of 1 in 100,000 . The symptoms resolve promptly with correction of potassium. The patient experiences motor symptoms while the sensation is preserved and can be differentiated from acute inflammatory demyelinating polyneuropathy with preserved ocular, bulbar or respiratory involvement.

\section{Case report}

A 23yr old man presented with generalised weakness of few hours duration. He woke up in the morning to find that he was unable to move his arms and legs. There was no respiratory distress. $\mathrm{He}$ experienced diarrhoea about twice a day for 2days which was about 3 days prior to his presentation. Neurological examination revealed reduced power 2/5 in all the limbs with hypotonia, hyporeflexia in all the joints and down going plantars. There was no sensory deficit. The respiratory rate was $16 / \mathrm{mt}$ and BP 136/75.

Bloods showed low potassium of 2.0 with normal sodium and other electrolytes. Blood gas showed an acidotic picture with a pH of 7.28 and $\mathrm{HCO} 3$ of 19 . ECG showed sinus tachycardia of $105 / \mathrm{m}$.

A spirometry could not be performed. The symptoms improved following IV administration of KCL.

\section{Discussion}

The mechanism is due to activation of $\mathrm{Na} / \mathrm{K} / \mathrm{ATP}$ ase pump leading to influx of potassium into the cells thus causing reduced serum potassium levels. Androgens activate the pump too, hence the condition is seen more commonly in men than women.

ECG changes in HPP include the changes usually seen in hypokalaemia which are prolonged PR interval, T wave flattening or inversion with ST depression, appearance of $U$ waves and QT prolongation.

Treatment includes potassium supplementation intravenously not faster than $10 \mathrm{mmol} / \mathrm{hr}$ to avoid rebound hyperkalaemia. Prompt treatment is mandatory as severe dyselectrolytaemia can cause asystole and cardiac arrest. Patients should be warned to avoid trigger factors and maintain adequate hydration. 